\title{
SAÚDE MENTAL NO CONTEXTO UNIVERSITÁRIO: DESAFIOS E PRÁTICAS
}

\begin{abstract}
Mental health in the university context: challenges and practices
\end{abstract}
La santé mentale dans le contexte universitaire: défis et pratiques

La salud mental en el contexto universitario: desafíos y práticas

Karine Vanessa Perez ${ }^{1}$

Université du Québec à Montréal, Montreal, Quebec, Canadá.

ORCID: https://orcid.org/0000-0003-1643-8042.

Psicóloga, Doutora e Mestre em Psicologia Social e Institucional pela Universidade Federal do Rio Grande do Sul (UFRGS). Pós-Doutorado (em andamento) na Université du Québec à Montréal. Docente da Universidade de Santa Cruz do Sul (UNISC). Integrante do Laboratório de Psicodinâmica do Trabalho (PPGPSI/UFRGS).

Luciana Gisele Brun ${ }^{2}$

Porto Alegre, Rio grande do Sul, Brasil.

ORCID: https://orcid.org/0000-0002-6622-2170

Psicóloga, Doutora em Psicologia Clínica na linha de pesquisa Processos de Saúde e Doença em Contextos Institucionais, com mestrado e graduação em Psicologia, MBA em Gestão de Pessoas pela Universidade do Vale do Rio dos Sinos - Unisinos e Formação em Dinâmica dos Grupos pela Sociedade Brasileira de Dinâmica dos

Grupos - SBDG.

Carlos Manoel Lopes Rodrigues ${ }^{3}$

Centro Universitário de Brasília, Brasília, Distrito Federal, Brasil.

ORCID: http://orcid.org/0000-0002-5188-7110

Psicólogo, Mestrado em Psicologia Social, do Trabalho e das Organizações (Psto) pela Universidade de Brasília (2018). Professor Adjunto do - UNICEUB na área de Avaliação Psicológica e Psicodiagnóstico.

\section{Resumo}

Este artigo busca contextualizar as questões relativas à saúde mental no contexto universitário, apresentando algumas práticas já desenvolvidas, além de citar desafios futuros. Discute tanto o sofrimento de estudantes quanto de professores no meio acadêmico. Foi produzido a partir de reflexões, bem como referências já publicadas a respeito da temática. Também cita brevemente os artigos que são apresentados neste dossiê, com o intuito de encontrar pontos em comum. Pode-se dizer que os estudos que envolvem o sofrimento psíquico no ambiente universitário são recentes e que esta problemática vem afetando tanto instituições públicas quanto privadas. Buscase propor alternativas à esse sofrimento com o intuito de facilitar o processo acadêmico de universitários e as vivências de professores no âmbito do trabalho nas instituições de ensino superior.

Palavras-chave: Saúde Mental; Sofrimento Psíquico; Ensino Superior; Estudantes Universitários; Professores Universitários.

\begin{tabular}{l}
\hline Abstract \\
\hline${ }^{\mathbf{1}}$ karinevanessaperez@gmail.com \\
${ }^{2}$ lubrun@gmail.com \\
${ }^{3}$ prof.carlos.manoel@gmail.com
\end{tabular}


This article seeks to contextualize the issues related to mental health in the university context, presenting some practices already developed, in addition to citing future challenges. It discusses both the suffering of students and teachers in the academic environment. It was produced from reflections, as well as references already published on the subject. It also briefly mentions the articles that are presented in this dossier, in order to find common ground. It can be said that the studies that involve psychological suffering in the university environment are recent and that this problem has been affecting both public and private institutions. It seeks to propose alternatives to this suffering in order to facilitate the academic process of university students and the experiences of teachers in the field of work in higher education institutions.

Keywords: Mental Health; Psychic Suffering; Higher Education; University Students; University Teachers.

\section{Résumé}

Cet article cherche à contextualiser les questions liées à la santé mentale dans le contexte universitaire, en présentant certaines pratiques déjà développées, en plus de citer les défis futurs. Il traite à la fois de la souffrance des élèves et des enseignants dans l'environnement académique. Il a été produit à partir de réflexions, ainsi que de références déjà publiées sur le sujet. Il mentionne également brièvement les articles qui sont présentés dans ce dossier, afin de trouver un terrain d'entente. On peut dire que les études sur la souffrance psychique en milieu universitaire sont récentes et que ce problème touche aussi bien les institutions publiques que privées. Il cherche à proposer des alternatives à cette souffrance afin de faciliter le processus académique des étudiants universitaires et les expériences des enseignants dans le domaine du travail dans les établissements d'enseignement supérieur.

Mots clés:Santé Mentale; Souffrance Psychique; Enseignement Supérieur; Étudiants Universitaires; Enseignants Universitaires.

\section{Resumen}

Este artículo busca contextualizar los temas relacionados con la salud mental en el contexto universitario, presentando algunas prácticas ya desarrolladas, además de citar desafíos futuros. Discute tanto el sufrimiento de los estudiantes como el de los profesores en el entorno académico. Se elaboró a partir de reflexiones, así como de referencias ya publicadas sobre el tema. También menciona brevemente los artículos que se presentan en este dossier, con el fin de encontrar una base común. Se puede decir que los estudios que involucran el sufrimiento psicológico en el ámbito universitario son recientes y que este problema ha estado afectando tanto a las instituciones públicas como a las privadas. Busca proponer alternativas a este sufrimiento para facilitar el proceso académico de los estudiantes universitarios y las experiencias de los profesores en el campo del trabajo en las instituciones de educación superior.

Palabras clave: Salud Mental; Sufrimiento Psíquico; Educación Superior; Estudiantes Universitarios; Profesores Universitarios

\section{INTRODUÇÃO}

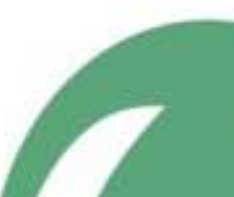

A proposta deste artigo é apresentar algumas reflexões a respeito da temática da saúde mental no meio universitário a partir de diferentes estudos produzidos por pesquisadores nacionais e internacionais. Parte de acontecimentos recentes nas universidades públicas e privadas para então apresentar algumas propostas de intervenção que vem sendo pensadas em algumas instituições de ensino superior. Uma compilação em dois volumes que agrega estudos teóricos, empíricos e relatos de experiência sobre esta temática emergente que vem chamando a atenção dos pesquisadores devido ao aumento do adoecimento mental e do suicídio de alunos e professores. 
Para ilustrar esse contexto nos utilizamos do testemunho de uma pós-graduanda sobre a sua experiência na pós-graduação e que foi enviado a Folha de São Paulo. Trata-se de uma estudante de Ciências Sociais da Universidade Federal de Juiz de Fora e que faz um relato sobre como tem sido sua vivência nos anos passados na universidade.

No meu mestrado, tive síndrome do pânico e achei que não ia conseguir terminar". Com apoio psicológico da universidade consegui concluir, apesar do péssimo relacionamento com minha orientadora que não entendia que estava doente. Cinco anos depois da defesa a minha tese continua jogada na estante e não consigo sequer olhar para ela. Entrei no doutorado, mas acabei desistindo. Hoje estou bem com essa escolha, pois o meio acadêmico não é para pessoas sensíveis. (https://www1.folha.uol.com.br/ciencia/2017/12/1943862-estudantes-de-mestrado-edoutorado-relatam-suas-dores-na-pos-graduacao.shtml, recuperado em 05 de dezembro, 2019).

Relatos semelhantes a este não são incomuns e tem sido manifestados por estudantes de diversas áreas de formação, nos mais variados estados brasileiros e também em outros países, conforme poderá ser visto de modo detalhado nos artigos apresentados por este dossiê. Para introduzir a discussão é importante contextualizar de maneira breve as questões sociais e econômicas que perpassam a vida nas universidades nos últimos anos.

O ensino superior brasileiro, tanto público quanto privado, passou por um período de expansão, especialmente a partir do início dos anos 2000, sendo que as dificuldades dos professores estavam relacionadas a tentativas de dar conta de tanta demanda de trabalho em função do grande número de alunos que ingressavam nas universidades (Cogo, 2008; Perez, 2012). Hoje temos o mesmo problema, porém colocado de outro modo, já que temos o encolhimento do número de docentes em detrimento da diminuição de estudantes. Alguns cursos, entre as licenciaturas por exemplo, possuem uma baixa procura, enquanto outros cursos são demasiadamente disputados, demandando um altíssimo empenho dos candidatos e dos alunos. Mudanças na demanda de oferta e procura de profissionais no mercado de trabalho que estão diretamente relacionadas com o ensino superior.

Não há como negar que este debate é urgente e deve ser feito articulando diversas instituições envolvidas no campo da educação e da saúde, especialmente as universidades e as políticas de saúde mental. Trata-se de um problema que diz respeito a saúde coletiva, não sendo questões que devem ser solucionadas individualmente somente. Há necessidade de um engajamento de estudantes, professores e profissionais que atuam no ensino superior, além dos próprios profissionais da saúde. Buscar melhores condições para o desenvolvimento da pesquisa, lutando contra o modelo vigente produtivista é também uma tarefa institucional. 


\section{APRESENTAÇÃO DO DOSSIÊ}

O que fazer diante deste contexto? É essencial o questionamento sobre como resistir e produzir outros modos de existir no contexto universitário que não obrigue os atores envolvidos a se sujeitar a lógica perversa do capital cada vez mais flexível e que não os faça sucumbir ao adoecimento coletivo que este contexto social os tem empurrado.

Neste sentido este dossiê busca lançar luzsobre esta temática, oportunizando reflexões e compartilhando iniciativas que já têm sido realizadas em diversos contexto universitários. Busca-se assim não oferecer "modos de fazer" e sim inspirar outras ações voltadas à saúde daqueles que estudam e trabalham nas instituições de ensino superior brasileiras e estrangeiras.

A leitura deste dossiê possibilitará observar que há artigos que versam sobre as vivências de professores universitários enquanto outros focam nos estudantes. Ambas as propostas buscaram contextualizar o ensino superior de modo geral, mas também procuraram localizar as ações relativas às universidades estudadas. As transformações no mundo do trabalho que tangenciam a vida nas instituições de ensino superior, também foram discutidas, buscando articular como o contexto econômico, político e social afetam universitários e trabalhadores da educação.

Os estudos que focam a vida dos professores universitários no meio acadêmico abordaram temáticas como: adoecimento psíquico e uso de substâncias psicoativas e a relação entre o processo e as condições de trabalho e o surgimento de sofrimento mental. Já os artigos que tratam da saúde mental dos estudantes abordaram os problemas físicos, psicológicos e sociais causados pela vida acadêmica; sintomatologia de estresse; saúde mental a partir de uma experiência de estágio curricular obrigatório; relato sobre projeto de extensão que fez uso de treinamento para promover a saúde mental dos estudantes; descrição a respeito de um serviço de atendimento psicológico com foco no sofrimento de estudantes. Além disso, há um artigo que aborda o trabalho de servidores de uma Universidade Federal do Brasil, que investigou os afastamentos para tratamento da saúde por transtornos mentais, ocasionados por questões que envolvem o trabalho.

Foram utilizados tantos métodos qualitativos como entrevistas semestruturadas, e encontros em grupo; quanto quantitativos como consulta em banco de dados, além de instrumentos como Alcohol, Smoking and Substance Involvement Screening Test e Beck Depression Inventory; Self Reporting Questionnaire (SRQ-20), Protocolo de Avaliação dos Riscos Psicossociais no Trabalho - PROART; Inventário de Sintomas de Stress de Lipp. Em 
alguns casos utilizou-se métodos associados (quali-quanti), bem como pesquisa-intervenção a partir da realização de treinamentos e escuta psicológica.

\section{Mundo do trabalho e saúde mental nas universidades: elementos para o debate}

O que se vive hoje na universidade como professores é a precarização do trabalho docente em que a sujeição impera. Não incomum são as demissões em massa ocorridas em inúmeras universidades privadas do Brasil, especialmente após a implementação das Reforma Trabalhista que ocorreu em novembro de 2017 (Antunes \& Praun, 2019).

Outro aspecto que vem transformando o ensino privado universitário é a educação à distância, alavancado pela amplificação do uso das plataformas educacionais virtuais (Valente, 2003). Tais recursos prometem, por um lado, oferecer maior acessibilidade ao ensino superior aliado ao menor custo e a maior flexibilidade de horários. Estas promessas vêm atraindo muitos alunos que buscam estes benefícios. bem como conciliar a jornada entre o trabalho e os estudos num cenário nacional competitivo e de grande desemprego. Por outro lado, as instituições privadas vêm modificando seus quadros funcionais, visando enxugar custos, bem como engajálos neste novo sistema de ensino. Resultando em constantes mudanças na organização do trabalho e tensão nas relações psicossociais entre colegas.

Tais fatores têm produzido um clima de medo, silêncio, ameaça e solidão nas instituições de ensino que tem gerado mal-estar não só no público docente (Monteiro, Brun, Santos, Tundis \& Cordon, 2019), como também tem repercutido na vida dos estudantes, seja na graduação, seja na pós-graduação, em que as relações ficam estremecidas e atravessadas pelo sentimento de insegurança que paira no ar das universidades, sejam públicas ou privadas, mas não só delas... do nosso país como um todo, em especial permeando a vida daqueles que vivem do trabalho (Antunes, 1995; 1999).

Dejours $(1992 ; 1999)$ estudioso da relação entre trabalho e dimensão psíquica, diz em suas obras que o que tem prevalecido nos últimos anos é a lógica do "cada-um-por-si", onde os colegas são vistos como inimigos e não como aliados na luta contra o sofrimento no trabalho. Hoje, vivenciando tantas perdas de direitos no contexto brasileiro, com a constante fragilização das políticas públicas, podemos afirmar que a lógica que prevalece é a do "salve-se quem puder". Quem tem um emprego, luta para mantê-lo, quem não tem busca qualquer atividade remunerada, seja ela registrada ou não. 
Diante deste quadro temos então um terreno propício para o desenvolvimento das patologias sociais e as patologias da solidão (Ferreira, 2007) encontram possibilidades de se alastrar. As patologias sociais são resultado da vivência de dificuldades extremas de dar conta das demandas exigidas pelo trabalho contemporâneo.

$\mathrm{Na}$ busca por conciliar as adversidades e o sofrimento gerado por elas pode desencadear um processo de anestesiamento e insensibilidade diante da dor e do sofrimento do outro, bem como de seu próprio sofrer. Junto a isso observa-se uma falência dos coletivos de trabalho, dos movimentos dos trabalhadores. E mais fundo ainda, e talvez consequentemente, a falta da confiança e da cooperação entre os trabalhadores. Neste sentido, percebe-se também a falta de cooperação, confiança também no universo acadêmico? Como tem se constituído os espaços para trabalhar e realizar pesquisa no contexto brasileiro? Ficam as reflexões...

Gaulejac, sociólogo francês que estuda os efeitos nefastos dos modelos de gestão na saúde dos trabalhadores, vai nos trazer o conceito de quantofrenia para referir-se a obsessão por números. Nas palavras do sociólogo "designa uma patologia que consiste em querer traduzir sistematicamente os fenômenos sociais e humanos em linguagem matemática" (Gaulejac, 2007, p. 94). Assim como pode-se notar a quantofrenia nas relações acadêmicas? As cobranças que estudantes e docentes são expostos dizem respeito a uma exigência numérica essencialmente, em que a quantidade é elegida em detrimento da qualidade do trabalho desenvolvido.

Além destas provocações é importante o questionamento sobre a sociedade do desempenho que parece ter se tornado a sociedade do cansaço. Para abordar esse ponto as ideias de um filósofo sul-coreano Byung-Chul Han (2015), que denuncia de modo exemplar as relações que estão se construindo atualmente. Ele nos diz que, hoje, as pessoas convivem com o sentimento de angústia por terem a sensação de que não estão fazendo tudo o que poderiam. Ou seja, se não for um vencedor, a culpa é do próprio sujeito. É comum atualmente a pessoa explorar a si mesma achando que está se realizando. Isso segue uma lógica traiçoeira do neoliberalismo que leva ao esgotamento.

Este é um sentimento que permeia não só os espaços de trabalho, mas também o espaço da formação profissional. Sendo assim, percebe-se a universidade como o lugar da educação, da formação profissional e da vida em comum que tem uma grande interface com o mundo do trabalho. Assim, fica o questionamento: a universidade não estaria também inserida NO mundo do trabalho, paradoxalmente lugar de crítica e, por vezes, reprodução daquilo que prescreve o sistema capitalista? Esse modo de produção, também produtor de subjetividade, de maneiras por vezes imperceptíveis, não teria também controlado a vida nas universidades fazendo com que as instituições de ensino incorporem a lógica produtivista do capitalismo contemporâneo? 
Observa-se que o aluno do ensino superior, muitas vezes, também concilia um trabalho, uma família e uma vida social, equilibrando seu tempo e suas economias. Isso é desafiador, especialmente, em função da redução drástica de políticas estudantis que visam a permanência de estudantes pobres nas universidades (Fundo de Financiamento Estudantil - FIES, por exemplo). Todo esse contexto tem se mostrado como potencial para desencadear o sofrimento e adoecimento mental no meio universitário.

Graner \& Cerqueira (2019) realizam uma revisão de literatura a partir de dados empíricos encontrados nas bases de dados Web of Science, Medline e Scopus com o intuito de identificar os principais fatores de proteção para o sofrimento psíquico em universitários. Chegaram à conclusão de que a maior frequência de estudos com este enfoque tem se dado nos cursos da saúde. Características da vida acadêmica e relacional foram os aspectos mais associados à presença de sofrimento psíquico em universitários. Fatores como violência no ambiente universitário, trote, bullying, discriminação social, além de estruturas pedagógicas e curriculares dos cursos podem causar efeitos negativos na saúde dos estudantes universitários.

Mesmo sendo muitas vezes ignorado, este fenômeno tem sido investigado e as informações que tais pesquisas têm revelado são preocupantes: quase metade dos estudantes da pós-graduação do Brasil e do mundo estão vivenciando situações de sofrimento e adoecimento psíquico. Este estudo refere-se a uma pesquisa internacional publicada na revista Nature que foi realizada em 26 países com 2.279 pós-graduandos. Quando comparados com a população em geral os estudantes de pós-graduação tem seis vezes mais probabilidade de vivenciar ansiedade e depressão (Evans, Bira, Gaztelum, Weiss \& Vanderford, 2018).

Neste contex to podemos citar os quadros ligados a ansiedade e depressão, que em alguns casos podem levar ao comportamento suicida. Para termos uma ideia, na Universidade de Brasília foram $11^{4}$ suicídios de estudantes no período de janeiro a julho de 2018. Evans et al., (2018) indica que $36 \%$ dos alunos de mestrado e doutorado sofrem de depressão moderada ou severa em comparação à $6 \%$ da população geral. Outra pesquisa da UFRJ de 2009 demonstra que 58\% dos estudantes apresentam níveis médios e altos de estresse. Outro estudo nacional da ANDIFES (Associação Nacional dos Dirigentes das Instituições Federais de Ensino Superior) de 2016 informa que 30\% dos estudantes de graduação em instituições federais do Brasil procuraram atendimento psicológico durante o período da formaçấo. Tais pesquisas ainda indicam uma maior prevalência desse sofrimento em mulheres, pessoas negras, estrangeiras ou

\footnotetext{
${ }^{4}$ Informações relatadas por profissionais da saúde que atuam em serviços de apoio ao estudante da Universidade de Brasília em um evento realizado pelo Conselho Regional de Psicologia do Distrito Federal (CRP-DF) em julho de 2018.
} 
trans. Entretanto, ainda existe uma carência de pesquisas que possam delinear melhor tais aspectos como raça, gênero, idade, nacionalidade e classe (https://saude.estadao.com.br/noticias/geral,nas-federais-3-estudantes-em-cada-10-ja procuraram-psicologo,70002003617, recuperado em 11 de dezembro, 2019).

Mas o que torna esses dados tão alarmantes? O que está se vivendo nas universidades hoje que poderia estar associado a tal sofrimento? Atualmente há uma maior pressão por resultados, quantifica-se o trabalho como nunca na história visto. Em relação ao contexto universitário há uma ideia de que a qualificação vai garantir melhores condições de trabalho e que a vida intelectual não estar suscetível à precarização do trabalho, o que de fato não acontece. As vivências da graduação e da pós-graduação são permeadas de expectativas.

A formação de nível superior também é percebida como um momento de transição entre a vida estudantil e a vida profissional o que coincide com sentimentos de insegurança e sentimento de incerteza sobre o futuro. Nem sempre o aluno encontra apoio em suas redes, sejam elas familiares, sociais ou até mesmo entre os colegas na universidade. Não dar conta das demandas impostas e não suportar a pressão do contexto são fatores entendidos como fraqueza e, de certo modo, como uma inabilidade para a carreira acadêmica. Decidir não trabalhar na pesquisa, ler e estudar no tempo livre é visto com estranheza pelos pares como se este sujeito que assim o faz fosse "folgado, preguiçoso e displicente" com os estudos e com a sua carreira. Ignora-se o fato de que as pausas são essenciais para o bom andamento do trabalho e necessárias para a saúde desses estudantes/profissionais.

Abordando a questão do sofrimento no espaço universitário pode-se fazer uso das palavras da pesquisadora Rosana Pinheiro Machado, cientista social e antropóloga que discute este tema, nos dizendo: "Não é raro que orientadores e estudantes abordem o assunto [sobre o sofrimento e a depressão na pós-graduação] de forma leviana, por meio de brincadeiras sutis que dão a entender que depressão é "frescura" ou "fraqueza", já que os "bons" teriam que “segurar o tranco". Estar na pós-graduação seria um privilégio e, por isso, todos deveriam estar contentes. Há também aqueles que romantizam o sofrimento mental como uma espécie de um ethos intrínseco às trajetórias dos gênios confusos, dos intelectuais desajustados ou do alto esforço do mérito." Ideias como esta, como bem exposto pela pesquisadora, estão presentes no cotidiano dos pós-graduandos. Ela continua, dizendo que "Esse tipo de visão sobre a dor e a ansiedade é perversa, irresponsável e ignorante de um quadro de alerta global cujo desenrolar mais drástico e irremediável é o suicídio.”, alertando para o quanto deve-se estar atentos aos fatores que têm produzido esse sofrimento e pensando em como transformar esse meio que 
pode ser sim adoecedor (https://saude.estadao.com.br/noticias/geral,nas-federais-3-estudantesem-cada-10-ja-procuraram-psicologo,70002003617, recuperado em 12 de dezembro, 2019).

\section{PARA FINALIZAR...}

Pontualmente, propor espaços de acolhimento aos estudantes são medidas importantes para que estes percebam que não estão sós e que tem apoio de pessoas qualificadas e de colegas que também passam por situações semelhantes. Assim como para ressignificar o sofrimento vivido nos espaços de trabalho, a função do coletivo tem um lugar importante. Desse modo, o acolhimento em grupo pode ser uma ferramenta a ser pensada, já que o sofrimento do trabalho e dos espaços de formação para o trabalho é produzido pelo contexto social em que estamos inseridos e desse modo deve ser "tratado".

De qualquer maneira, falar sobre o tema, seja em encontros, seja por meio da mídia e especialmente por meio das redes sociais, faz com que a população em geral tome conhecimento da problemática em questão. Ainda que a potência esteja em criar novos modos de existir e resistir a este modo de construirmos a vida acadêmica. Encontros, nos seus mais diferentes formatos, abrem espaço para discussões que não podem se encerrar aqui e que poderão semear novas possibilidades de habitar as universidades, indicando que outros modos de viver a academia precisam ser inventados, o que deve ser percebido como uma tarefa do coletivo.

\section{REFERÊNCIAS}

Antunes, R. (1995). Adeus ao trabalho? São Paulo: Cortez.

Antunes, R. (1999). Os sentidos do trabalho. São Paulo: Boitempo.

Antunes, R., \& Praun, L. (2019). A aposta nos escombros: reforma trabalhista e previdenciária - a dupla face de um mesmo projeto. Revista JuríDica Trabalho E Desenvolvimento Humano, 2(1). doi:10.33239/rtdh.v2i1.43

Cogo, P. S. (2008). Demissão: uma ruptura na trajetória profissional de professores do ensino superior privado. Porto Alegre: UFRGS. 227 p. Tese (Doutorado em Sociologia) Programa de Pós-Graduação em Sociologia, Universidade Federal do Rio Grande do Sul.

Dejours, C. (1992). A loucura do trabalho: estudo de psicopatologia do trabalho. São Paulo: Cortez-Oboré.

Dejours, C. (1999). Banalização da injustiça social. São Paulo: Fundação Getúlio Vargas. 
Evans, T. M., Bira, L., Gaztelum, J. G., Weiss, L. T., Vanderford, N. L. (2018). Evidence for a mental health crisis in graduate education: Evidence for a mental health crisis in graduate education. Nature Biotechnology;36(3):282- 284.

Ferreira, J. B. (2007). Trabalho, sofrimento e patologias sociais: estudo com trabalhadores bancários e anistiados políticos de uma empresa pública. Dissertação (Mestrado em Psicologia) - Universidade de Brasília, Brasília.

Gaulejac, V. de. (2007). Gestão como doença social: ideologia, poder gerencialista e fragmentação social. São Paulo: Ideias e Letras.

Graner, K. M., \&amp; Cerqueira, A. T. A. R. (2019). Revisão integrativa: sofrimento psíquico em estudantes universitários e fatores associados. Ciência \&amp; Saúde Coletiva, 24(4), 1327-1346. Epub May 02, 2019. https://dx.doi.org/10.1590/1413-81232018244.09692017

Han, B.-C. (2015). A sociedade do cansaço Petrópolis: Vozes.

Monteiro, J. K., Brun, L. G., Santos, A. S., Tundis, A., Cardon, S. B. (2019) Distúrbios Psiquiátricos Menores e Fatores Associados em Professores do Ensino Privado do Rio Grande do Sul/Brasil. Revista Contextos Clínicos 12, 3 (set/dez. 2019). doi: 10.4013/ctc.2019.123.07

Valente, J. A. (2003). Educação a distância no ensino superior: soluções e flexibilizações. Revista Interface - Comunic, Saúde, Educ, v7, n12, p.139-48. Disponível em https://www.scielosp.org/pdf/icse/2003.v7n12/139-142/pt

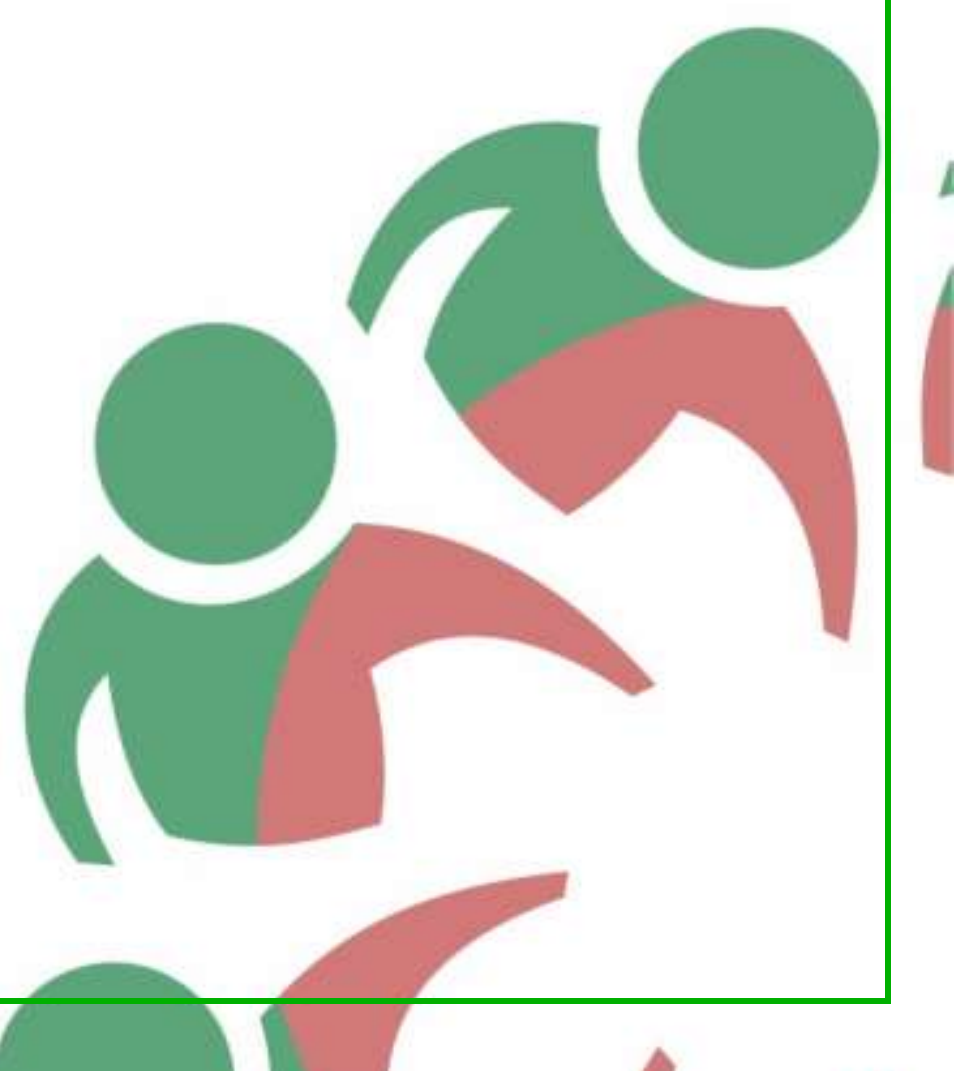

